\title{
The job to be done with biofilm and titanium implants
}

\author{
Ole T. Jensen ${ }^{1}$, Ervin Weiss ${ }^{2}$, David W. Grainger ${ }^{3}$ \\ ${ }^{1}$ Department of Oral Maxillofacial Surgery, University of Utah School of Dentistry, Salt Lake City, Utah, USA; ${ }^{2}$ Tel Aviv University School of Dental \\ Medicine, Tel Aviv, Israel; ${ }^{3}$ Department of Bioengineering, University of Utah School of Dentistry, Salt Lake City, Utah, USA \\ Correspondence to: Ole T. Jensen. Department of Oral Maxillofacial Surgery, University of Utah School of Dentistry, Salt Lake City, Utah, USA. \\ Email: oletjenen@icloud.com.
}

Received: 23 April 2021; Accepted: 31 May 2021; Published: 10 September 2021.

doi: 10.21037/fomm-21-54

View this article at: http://dx.doi.org/10.21037/fomm-21-54

Once, Clayton Christensen was asked about the prospect of starting a new dental implant company when there were already hundreds of implant companies around the world. "If you proceed," he advised, "you have to ask one question: What is the job to be done?" Interestingly, the job to be done remains one that the dental profession has wrestled with from the beginning: to inhibit or arrest the development of microbial biofilms on titanium surfaces of an intraoral implant.

The peculiarity of the oral cavity is that endogenous bacteria and fungus - commensal, pathogenic, opportunistic - all evolved a highly stable self-regulating, and often symbiotic, environment for the dentate niche, termed biofilm that supplements their planktonic growth strategy. A biofilm is a community of aggregated microbial cells organized as micro-communities, colonizing solid oral surfaces in contact with liquids and air. And this unique biofilm strategy is several orders of magnitude more resistant to natural sheer forces from deglutition, mastication and salivary flow that otherwise readily clear nonadherent pathogens from the mouth.

The biofilm matrix comprises an aqueous network of mixed nucleic acids, polysaccharides, proteins and lipids, all of microbial origin. The interacting extracellular polymeric substances (EPS) are non-covalently associated into a robust matrix to embed and protect aggregated bacterial and fungal cells within the biofilm. Microbe-microbe, microbe-EPS, microbe-liquid/air, and microbe-substrate interactions all determine formation, properties and behaviors of biofilm. Polymicrobial biofilms are most common, representing complex dynamic communities of diverse, spatially aggregated organisms.

One characteristic feature of biofilm is its physical barrier functions that provide microbial protection, particularly in the deeper layers. Biofilm protections are diverse, spanning microbial physical resistance to phagocyte engulfment and biofilm extraction, and reduced exposure to antimicrobials by limited biofilm permeation. Additionally, microbial density within EPS highly favors plasmid exchange, facilitating the transfer of resistance genes and virulence factors. Other genetic programming and regulation also occurs within biofilms, allowing populations of pathogens to undergo senescence to avoid susceptibility to metabolically targeted antimicrobials. Sleeper or persister cells re-awaken post-exposure to exert virulence. Furthermore, should the biofilm become mechanically or pharmacologically disrupted, they readily and rapidly reform in the oral cavity within several hours. Biofilm, therefore, is highly refractory to elimination from the oral niche. Importantly, commensal and probiotic endogenous oral biofilms are an essential component of oral health and therefore should not be disturbed. Opportunistic, pathogenic oral biofilms are a source of oral disease and niche compromise. Despite highly disparate contributions to oral health and demise, all biofilms are structurally and biologically integral to the oral environment. Effective mitigation and selective neutralization of pathogenic oral biofilms while preserving and promoting commensal host-beneficial oral biofilms is therefore the job to be done. Restoring and maintaining a probiotic balance in promoting healthy biofilms as a defense against pathogenic biofilms is an essential goal.

Natural teeth re-implanted into alveolar bone and invested with supportive soft tissue attachment are, to a degree, self-cleansing, and when teeth and jaw structures are aligned, promote a type of stand still equilibrium with biofilm. But this scenario can become imbalanced 
by a change in the oral structural basis, such as is found with a dental implant-supported fixed denture, a change in diet such as high sucrose intake, immune compromise as common in older patients who suddenly develop peri-implant disease, or disturbance in vascular dynamics associated with radiation therapy or prolonged bisphosphonate use.

Keeping oral biofilm at bay is an impossibility, and likely also counter-productive to the essential niche benefits of healthy oral biofilms. New physicochemical strategies are continuously introduced to augment the present treatment using oral prophylaxis, titanium surface decontamination and diverse assortments of novel topical or systemic bioactive strategies.

The canonical stages of biofilm formation, including microbe attachment, maturation and dispersion, are often a focus in producing methods to address biofilm through disrupting one or more of these stages. Microbial surface attachment (i.e., adhesion) is foundational; floating biofilms are possible but likely of less impact under continuous flushing of the oral cavity. Attachment disruption is therefore often posited as the sine qua non in combating biofilm. A central basic principle of biofilm surface mitigation is the predominant negatively charged membranes of most microbes, and repulsion by surfaces that are also negatively charged. Both mucosa and endothelium are such natural living surfaces. Zirconium and titanium oxides, stainless steel, and some synthetic polymers exhibit negative surface charge density when pristine. Other biomaterials develop negative charges naturally in physiological milieu as a consequence of adsorption of various components (e.g., lipids, proteins, ions) from physiological fluids that impart negative charge. This surface conditioning film begins within microseconds of implantation and evolves over time. Local dynamics between biomaterials adsorptive conditioning by oral fluids, continuous washing by food bolus, ingested microbes, and ambient air all contribute to the implant surface colonized by biofilm. This conditioned surface film covering the original biomaterials surface is often very different than the pristine implant surface.

All of the various methods used to prevent or reduce biofilm contamination of oral endoprostheses do not prevail under such continuous adsorptive and adhesive circumstances. All generally and ultimately fail to control biofilm with additional inputs (mechanical dental hygiene/ cleansing, antiseptic washes, and probiotic maintenance), and even then, most interventions only delay ultimate implant colonization. Improved technological advances are constantly published. New dental materials such as antimicrobial composite restoratives used to treat dental caries claim to be bactericidal long-term, using surface contact killing chemistry to help prevent secondary caries that otherwise occurs more than $50 \%$ of the time after 5 years of restoration. Dental implants, with an incidence of $>10 \%$ of significant peri-implant disease within several years of placement cannot so easily be removed and replaced like at failed dental filling. Reliable biofilm-prevention strategies represent an unmet need for all dental implant materials.

As host tolerance and immunological mechanisms accommodating implants change in relation to health, age and even local circumstances such as periodontal infection of an adjacent tooth, new circumstances compound risks to destabilizing osseointegration, leading to exposure of the implant surface, frequently titanium. These physiological changes can suddenly emerge to alter the risk profile, produce new host-biofilm dynamics in the oral cavity at the implant site, and necessitate enhanced oral hygiene measures to keep deleterious biofilm effects from compromising the dental device.

Continual exposure of an implant surface to the oral environment is speculated to be from $2 \%$ to $30 \%$ by ten years in function. Causes are often proposed to be physiologic, but more often are related to practitioner error such as poor surgical placement or inadequate prosthetic management such as use of misfit components, poorly executed restorations, or restorations designed with compromised cleanability. Of course, if clinician mistakes are not made and patient compliance is circumspect, a steady state is commonly maintained despite a trans-osseous foreign body penetrating into a biofilm-laden oropharynx. But dental implant mistakes are often made and patients often do not comply with preventive hygiene measures. And other health-related changes can occur over time as well, including physiological, pharmacological or ingestionrelated insults (e.g., tobacco smoking) that lead to catabolic bone changes at the bone-implant interface.

One replacement implant study showed a mean late timeframe for implant loss of 11 years, implying exposure of implant surfaces over time to oral biofilms with attendant loss of osseointegration. In certain patients, once an implant surface is exposed, biofilm appears to accelerate further implant surface exposure over time. Therefore, device surface modification using pharmacological and/or antimicrobial or probiotic materials and coatings is often used to mitigate implant failure risks that for myriad reasons 
become exposed and biofilm-colonized in order to curtail or even arrest progression of peri-implant disease.

This volume addresses loss of hard tissue attachment and issues with bacterial biofilm from the perspective of a variety of aspects: from risk assessment and oral hygiene measures to implant salvage, implant removal and jawbone reconstruction. Addressing the question of what to do at any one stage in time with these challenges is clinically significant for what appears to be a growing problem in a profession hampered by lack of consensus on what constitutes treatable peri-implant disease, what controlled studies concerning marginal bone loss really mean, what the definition of peri-implant disease actually is, and lack of implant-centered options to modulate ubiquitous oral biofilms to improve oral health and implant functional longevity.

\section{Acknowledgments}

Funding: None.

\section{Footnote}

Provenance and Peer Review: This article was commissioned by the editorial office, Frontiers of Oral and Maxillofacial Medicine for the series "Current Advances in Treatment of Peri-Implantitis". The article did not undergo external peer review.

Conflicts of Interest: All authors have completed the
ICMJE uniform disclosure form (available at https:// fomm.amegroups.com/article/view/10.21037/fomm-2154/coif). The series "Current Advances in Treatment of Peri-Implantitis" was commissioned by the editorial office without any funding or sponsorship. OTJ served as the unpaid Guest Editor of the series and serves as an unpaid editorial board member of Frontiers of Oral and Maxillofacial Medicine from Dec 2019 to Nov 2021. OTJ serves as the Chairman of Ditron Dental USA LLC, and the vice chairman of $\mathrm{Nobio}^{\mathrm{tm}}$ LTD. EW serves as one of the inventors of an antibacterial technology currently licensed to Nobio ${ }^{\text {tm }}$ LTD. Kadima Israel and also the founder of the company, Chief Scientist, shareholder, and executive board member. The authors have no other conflicts of interest to declare.

Ethical Statement: The authors are accountable for all aspects of the work in ensuring that questions related to the accuracy or integrity of any part of the work are appropriately investigated and resolved.

Open Access Statement: This is an Open Access article distributed in accordance with the Creative Commons Attribution-NonCommercial-NoDerivs 4.0 International License (CC BY-NC-ND 4.0), which permits the noncommercial replication and distribution of the article with the strict proviso that no changes or edits are made and the original work is properly cited (including links to both the formal publication through the relevant DOI and the license). See: https://creativecommons.org/licenses/by-nc-nd/4.0/. doi: 10.21037/fomm-21-54

Cite this article as: Jensen OT, Weiss E, Grainger DW. The job to be done with biofilm and titanium implants. Front Oral Maxillofac Med 2021;3:21. 